\title{
Infrasound induces coronary perivascular fibrosis in rats
}

\author{
Ana Lousinha a,*, Maria João R. Oliveira ${ }^{\mathrm{b}}$, Gonçalo Borrecho ${ }^{\mathrm{a}}$, José Brito ${ }^{\mathrm{a}}$, Pedro Oliveira a ${ }^{\mathrm{a}}$, \\ António Oliveira de Carvalho ${ }^{c}$, Diamantino Freitas ${ }^{c}$, Artur P. Águas ${ }^{b}$, Eduardo Antunes ${ }^{a}$ \\ a Center for Interdisciplinary Research Egas Moniz (CIIEM), Health Sciences Institute, Monte de Caparica, Portugal \\ ${ }^{\mathrm{b}}$ Department of Anatomy and UMIB, Abel Salazar Institute of Biomedical Sciences (ICBAS), University of Porto, Porto, Portugal \\ c Faculty of Engineering (FEUP), University of Porto, Porto, Portugal
}

\section{A R T I C L E I N F O}

\section{Article history:}

Received 20 September 2018

Received in revised form 27 September 2018

Accepted 3 October 2018

Available online $\mathrm{xxxx}$

\section{Keywords:}

Infrasound

Low-frequency noise

Coronary arteries

Fibrosis

Inflammation

\begin{abstract}
A B S T R A C T
Background: Chronic exposure to industrial noise is known to affect biological systems, namely, by inducing fibrosis in the absence of inflammatory cells. In rat hearts exposed to this environmental hazard, we have previously found myocardial and perivascular fibrosis. The acoustic spectrum of industrial environments is particularly rich in high-intensity infrasound $(<20 \mathrm{~Hz})$, whose effects on the heart are unknown. We evaluated the morphological changes induced by IFS in rat coronaries in the presence and absence of dexamethasone.

Methods: Adult Wistar rats were divided into three groups: group A (GA)-IFS ( $<20 \mathrm{~Hz}, 120 \mathrm{~dB}$ )-exposed rats for 28 days treated with dexamethasone; group $\mathrm{B}(\mathrm{GB})-\mathrm{IFS}$-exposed rats; group C (GC)-age-matched controls. The midventricle was prepared for observation with an optical microscope using $100 \times$ magnification. Thirty-one arterial vessels were selected (GA 8, GB 10, GC 13). The vessel caliber, thickness of the wall, and perivascular dimensions were quantified using image J software. Mann-Whitney and Kruskal-Wallis tests were used to compare the groups for lumen-to-vessel wall (L/W) and vessel wall-to-perivascular tissue $(\mathrm{W} / \mathrm{P})$ ratios.

Results: IFS-exposed rats exhibited a prominent perivascular tissue. The median L/W and median W/P ratios were 0.54 and $0.48,0.66$ and 0.49 , and 0.71 and 0.68 , respectively, in GA, GB, and GC. The W/P ratio was significantly higher in GC compared with IFS-exposed animals $(P=.001)$. The difference was significant between GC and GB $(P=.008)$ but not between GC and GA.

Conclusion: IFS induces coronary perivascular fibrosis that differs under treatment with corticosteroid.
\end{abstract}

(c) 2018 Elsevier Inc. All rights reserved.

\section{Introduction}

Noise represents a major environmental factor and is among the stressors with the highest impact on public health [1]. Noise and sound are physically the same, but the reaction to perception varies between people, depending on the cognitive environment in which detection takes place and ultimately leads to a definition of noise as an undesired sound $[2,3]$. Low-frequency noise (LFN) and infrasound (IFS) are conventionally defined as sound below 200 and $20 \mathrm{~Hz}$, respectively. The lower limit of the audio frequency range of human hearing is usually given as 16 or $20 \mathrm{~Hz}$, but humans can perceive infrasound if the sound pressure level $(\mathrm{dB})$ is sufficiently high [4]. In the range of IFS, comparative studies have shown that the auditory sensitivity of different species can vary widely. For instance, rats have poorer infrasonic

\footnotetext{
* Corresponding author.

E-mail addresses: alousinha@gmail.com (A. Lousinha), mjoliveira@icbas.up.pt (M.J. R. Oliveira), gborrecho@gmail.com (G. Borrecho), britojaa@hotmail.com (J. Brito), pedromaoliveira@hotmail.com (P. Oliveira), carvalho@fe.up.pt (A. Oliveira de Carvalho), dfreitas@icbas.up.pt (D. Freitas), aguas@icbas.up.pt (A. P. Águas), ejpantunes@sapo.pt (E. Antunes).
}

hearing than humans, considering different sound pressure levels [5], but high-intensity $(110 \mathrm{~dB})$ IFS vibrations on experimental rats can be perceived, as they elicit active avoidance reactions [6]. Beside its auditory health effects, noise can cause nonauditory effects-such as annoyance, sleep disturbance, and psychological stress-that experimental and epidemiological evidence links to cardiovascular disease, including ischemic heart disease, heart failure, arterial hypertension, arrhythmia, and stroke [7-12].

In recent years, scientists have directed their attention towards the relatively understudied noise range of below $200 \mathrm{~Hz}$. LFN and IFS are present everywhere, from natural occurrences to industrial installations and low-speed machinery. The characteristics of strong penetration and less attenuation in long distance propagation have been proposed to explain several adverse biological effects in experimental and epidemiological studies [13]. Low-frequency sounds have higher energy than the sounds at mid and higher frequencies and cannot be correctly evaluated using the conventional A-filters, which are most often used in environmental studies [14]. It is also possible that there are subtle effects of LFN on the body that we do not yet understand. High sound pressure levels $(>=90 \mathrm{~dB}$ ) of LFN can induce resonance responses in body cavities [13]. The overall range of human body resonant frequencies was 
found to be from 2 to $16 \mathrm{~Hz}$ [15], which is nearly the exact range of IFS. It may be assumed that animals also possess inherent specific sound frequencies in certain tissues and organs [16], and for that reason, it is important to document, using animal models, the morphological and biological effects induced by a wide spectrum of wavelengths, from industrial to LFN and IFS.

The cardiovascular system of rodents is sensitive to LFN [17-19]. We previously documented the development of perivascular fibrosis around the coronary arteries (from small to large caliber) of rats exposed to industrial noise $[20,21]$. We also found a significant fibrotic development in ventricular myocardium among rats submitted to LFN $[22,23]$. These morphological changes were found in the absence of inflammatory cells, which could suggest a noninflammatory process. However, the fibrotic proliferation mechanism remains unclear.

The effects of IFS on the coronary artery morphology under the influence of an anti-inflammatory agent are unknown. In order to fill this gap, we sought to evaluate the morphological changes induced by IFS in rat coronary arteries in the presence and absence of dexamethasone.

\section{Material and methods}

Fourteen adult female Wistar rats 10 months old were used in this study. They were purchased from a Spanish breeder (Charles River Laboratories España, S.A., Spain). All the handling and care of the experimental animals were performed by authorized researchers (accredited by the Federation of European Laboratory Animal Science Associations, Category C) and were done in accordance with the EU Commission on Animal Protection for Experimental and Scientific Purposes (2010/63/ $\mathrm{EU}$ ) and with the Portuguese legislation for the same purpose (Decree-Law No. 197/96). The rats were housed in $42 \times 27 \times 16-\mathrm{cm}$ polypropylene cages with a steel lid and had unrestricted access to food (commercial chow) and water. The same standard house conditions were used throughout the experiment for all the animals, and they involved keeping a maximum of two rats in a single cage.

In the beginning of the study, the 14 rats were randomly distributed into three groups. Nine of the rats were continuously exposed to highintensity and very LFN $(2-20 \mathrm{~Hz} / \mathrm{Lp}=114 \mathrm{~dB})$ during a period of 28 days. In four of the noise-treated rats, two tablets of dexamethasone $0.5 \mathrm{mg}$ (Decadron $0.5 \mathrm{mg}$, Medinfar) were introduced subcutaneously in the dorsal region at two time points of the noise exposure, day one and day 12 , and these were designated as group $A$, while the dexamethasone-free rats were included in group $B$. The remaining five rats were used as age-matched controls (group C) and sacrificed when all of the rats reached 11 months of age.

\subsection{Short description of electroacoustic experiment}

With the objective of creating a strong subsonic acoustic field in the vivarium chamber, a slightly trapezoidal room with $23.7 \mathrm{~m}^{3}$ $(3.55 \times 3.31 \times 2.02$, average length $\times$ width $\times$ height, respectively, in meters), a pseudo-random waveform in the $2-\mathrm{Hz}$ to $20-\mathrm{Hz}$ decade band was designed with Matlab based on a bandpass-filtered 30-s maximum length sequence segment. The waveform was used to excite an array of two infinite baffles mounted 18-in. 300-W-rated magnetodynamic subwoofers, by means of a $2 \times 600$-W heavy-duty quasi-dc voltage output audio power amplifier. Subsequently, with the aim of exploiting as much as possible the available subwoofers dynamic range at this frequency range with an acceptable amplitude distortion, the waveform was iteratively nonlinearly treated with moderate compressionexpansion and further filtering (in order to reduce the crest factor to approximately 2.0 times). The total sound pressure level and the spectral characteristics of the resulting acoustic pressure waveform were monitored, and the results were an average sound pressure level of $120 \mathrm{~dB}$ with a tolerance of $\pm 3 \mathrm{~dB}$ in the 30 -s time window. As to the spectral boundedness of the produced sound field, the result was $80 \mathrm{~dB}$ total out-of-band average sound pressure level ( $-40 \mathrm{~dB}$ lower).

\subsection{Light microscopy}

All rats were sacrificed by an intravenous injection of $0.6 \mathrm{ml}$ of a 5:4 mixture containing ketamine (Imalgene 1000, Bayer, Portugal) and xylazine (Rompun, Bayer, Portugal). The vascular system was perfused with a saline solution followed by paraformaldehyde fixation. The heart was excised, sectioned transversely from the ventricular apex to the atria, and routinely processed for light microscopy. The midventricular fragment from each heart was selected for the study. Five-micrometer paraffin-embedded slices of the tissue samples were made and dyed according to Sirius red techniques. The histological images were acquired with an optical microscope using $100 \times$ magnification.

\subsection{Histomorphometric data}

Thirty-one arterial vessels were selected ( 8 in GA, 10 in GB, and 13 in GC) (Fig. 1). At least one vessel from each rat was included. The researchers, including data collectors and data analysts, were blinded to which group the animals belonged to. Data were analyzed using the image J software (National Institutes of Health, Bethesda, MD, USA). The caliber of the arterial vessels, the thickness of the walls, and the perivascular tissue dimension were measured, and for each rat, the mean lumen-to-vessel wall (L/W) and mean vessel wall-toperivascular tissue (W/P) ratios were calculated (Fig. 2). (See Table 1.)

\subsection{Statistical analysis}

Mann-Whitney test has been applied in the comparison of IFS-exposed animals (including animals treated with dexamethasone and nontreated animals) and a control group for two parameters: L/W and W/P ratios. Kruskal-Wallis and Mann-Whitney tests were used in the comparison of the three groups for the same parameters. A $P$ value $<.05$ was considered statistically significant.

\section{Results}

\subsection{IFS-exposed animals vs. control animals}

The Mann-Whitney test has been used to compare the two groups for $\mathrm{L} / \mathrm{W}$ ratio and $\mathrm{W} / \mathrm{P}$ ratio variables, with the Bonferroni correction $\alpha^{*}=0.05 / 2=0.025$. The analysis shows that the W/P ratio is significantly lower in the IFS-exposed group $(P=.001)$. In contrast, the $\mathrm{L} / \mathrm{W}$ ratio did not differ between the two groups $(P=.060)$. It should be mentioned that the extreme observation for $\mathrm{W} / \mathrm{P}$ ratio values in the control group does not influence these conclusions, as differences between the groups were still detected by the Mann-Whitney test after removal of that observation $(P=.003)$, as expected in view of the robustness of this nonparametric test against such extreme values (Fig. 3).

\subsection{Comparison between IFS-exposed dexamethasone-treated animals, IFS-exposed animals, and control animals}

In the comparison between the three groups, the Kruskal-Wallis test has been applied with the same Bonferroni correction to the significance level, $\alpha^{*}=0.025$. The analysis has shown that there are differences between the groups for $\mathrm{W} / \mathrm{P}$ ratio $(P=.011)$ but not for $\mathrm{L} / \mathrm{W}$ ratio $(P=.104)$. Post hoc comparisons between the groups were conducted for $\mathrm{W} / \mathrm{P}$ ratio, using the Mann-Whitney test, at the $0.025 / 3=$ 0.0083 significance level to control for inflation of type 1 error. In this case, differences were detected between control and IFS-exposed animals not treated with dexamethasone $(P=.008)$. It should be mentioned that the extreme observation of $\mathrm{W} / \mathrm{P}$ ratio values does not seem to influence the main conclusion of the Kruskal-Wallis test, as expressed by a significance of .021 of the test result after removal of that observation, but it does change the conclusions of the Mann-Whitney test in the comparison between groups $\mathrm{B}$ and $\mathrm{C}$, which is now 


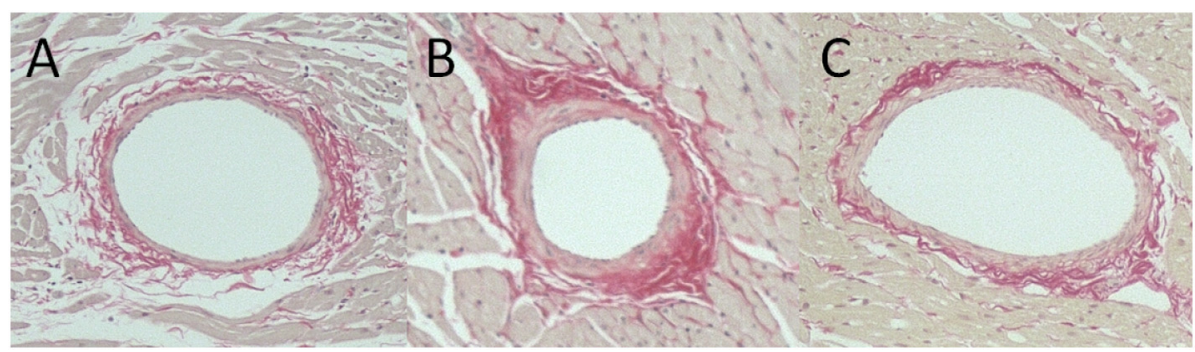

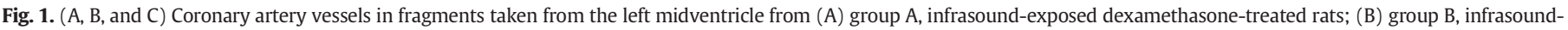
exposed rats; and (C) control group. Note the prominent perivascular tissue in infrasound-exposed animals [Sirius red, $100 \times$ ].

nonsignificant $(P=.021)$ under the Bonferroni correction $\left(\alpha^{*}=0.0083\right)$ (Fig. 4).

\section{Discussion}

The present study evaluated the coronary morphological changes in rat heart induced by pure IFS, created in a laboratory controlled electroacoustic experiment, and is the first study assessing the possible influence of an anti-inflammatory agent on these changes.

In this investigation, we found an increase in the perivascular tissue around the coronaries in rats exposed to IFS. There were significant differences between IFS-exposed rats and controls concerning the mean $\mathrm{W} / \mathrm{P}$ ratio, higher among the control group $(P<.001)$. But such differences did not reach statistical significance in the comparison between the animals treated with dexamethasone and the control group, pointing to a possible influence of this potent anti-inflammatory agent.

Previous work from our group, in Wistar rats, investigated the histomorphometric changes in the large and small coronary arteries induced by high-intensity industrial noise within a wide spectrum of wavelengths that included LFN, this last characterized by large sound pressure amplitude $\geq 90 \mathrm{~dB}$ and low-frequency bands of $\leq 500 \mathrm{~Hz}[20$, 21]. The exposure time ranged from 1 to 7 months. In both studies, we found the development of perivascular fibrosis in the absence of inflammatory cells, regardless of exposure time. In another study, we have

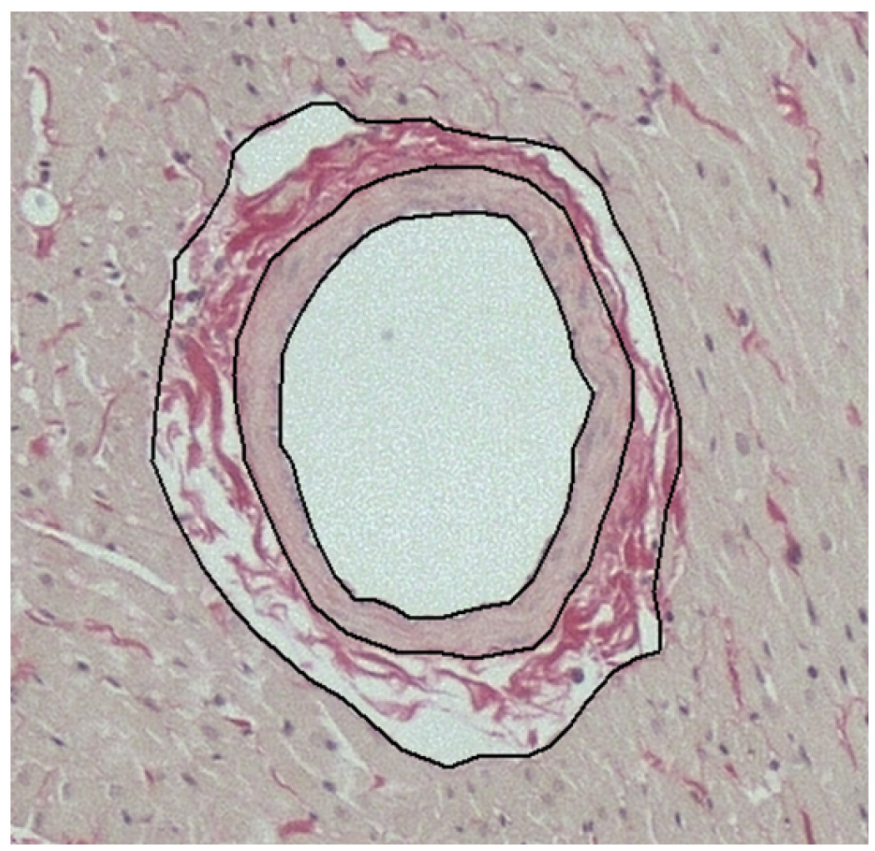

Fig. 2. Example of a coronary artery in a fragment taken from the left midventricle of an infrasound exposed rat [Sirius red, $100 \times$ ]. The black lines represent the measurements performed using Image $j$ software and correspond to vessel caliber, thickness of the wall, and perivascular dimension. These were used to calculate the $\mathrm{L} / \mathrm{W}$ and $\mathrm{W} / \mathrm{P}$ ratios. documented a significant fibrotic development in ventricular myocardium of rats exposed to LFN during a period of 3 months [22]. These investigations confirmed the abnormal proliferation of connective tissue as the main morphological change induced by LFN.

With increasing urbanization, noise is rising as one of the most important environmental risk factors in modern societies. The importance of the characteristics of the noise stimulus, such as frequency content, intensity, mean and peak dB level, pattern, and exposure time, is not well understood. In the quantitative risk assessment of environmental noise, the World Health Organization (WHO) Regional Office for Europe is concerned with sound pressure level limits, not frequencies [1]. Nonetheless, WHO also acknowledges the special place of LFN as an environmental problem, recognizing that the evidence is sufficiently strong to warrant immediate concern.

Sources of LFN include natural occurrences, industrial installations, and low-speed machinery, ranging from very low-frequency atmospheric fluctuations up to lower audio frequencies. Due to the characteristics of strong penetration and less attenuation in long distance propagation, it has been implicated in several adverse biological effects in experimental and epidemiological studies [13].

One effect of high pressure levels of LFN is excitation of body vibrations $[13,19,24]$. At high sound levels, typically above $80 \mathrm{~dB}$, the occurrence of resonance responses in body cavities was described [24]. The overall range of human body resonant frequencies was found to be from 2 to $16 \mathrm{~Hz}$ [15], which is almost the exact range of infrasound. The displacement between the organ and the skeletal structure places biodynamic strain on the body tissue involved, and it is known to reach its maximum under exposure to vibration close to the body's resonant frequency. Despite the practical impossibility of stimulating the natural frequency of one organ alone without exciting the whole-body resonances, measurements of vibration transmissibility from the point of excitation to a specific organ reveal frequencies of maximum transmissibility that can be attributed to the resonance of the organ. Considering that animals also possess inherent specific sound frequencies in certain tissues and organs [16], it is important to assess the morphological and biological effects induced by noise with different wavelengths in distinct animal models. So far, we have focused our investigation on the effects of large pressure amplitude noise within a wide spectrum of wavelengths, from the industrial to LFN and IFS, and with different exposure times, from 1 to several months [20-23]. The common finding was an abnormal deposition of collagen in the extracellular matrix (ECM), regardless of the characteristics of the noise stimulus other than pressure amplitude.

Table 1

Median (interquartile range) of the two measured outcomes in the three groups

\begin{tabular}{lll}
\hline & $\begin{array}{l}\text { Ratio L/W } \\
\text { Median (interquartile range) }\end{array}$ & $\begin{array}{l}\text { Ratio W/P } \\
\text { Median (interquartile range) }\end{array}$ \\
\hline Group A & $0.54(0.17)$ & $0.48(0.15)$ \\
Group B & $0.66(0.09)$ & $0.49(0.08)$ \\
Group C & $0.71(0.10)$ & $0.68(0.08)$ \\
\hline
\end{tabular}




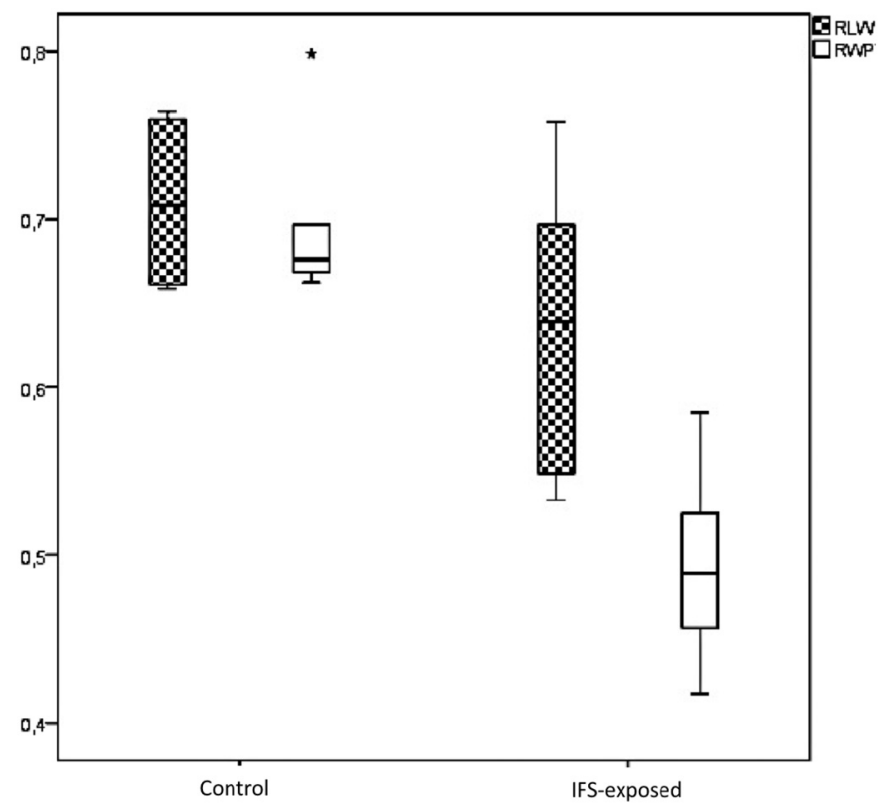

Fig. 3. Lumen-to-vessel wall and vessel wall-to-perivascular tissue ratios in IFS-exposed and control animals. The W/P ratio was significantly reduced in IFS-exposed animals ( $P=.001)$. RLW, lumen-to-vessel wall ratio; RWP, vessel wall-to-perivascular tissue ratio.

Interest in the potential adverse health effects of IFS has increased over time. High-level IFS below $20 \mathrm{~Hz}$ was historically thought to be of much less significance than LFN in the $20-200 \mathrm{~Hz}$ range at the same pressure level [25]. Research on the impact of IFS on the environment established that, for levels above $120 \mathrm{~dB}$, it is dangerous to the human body [13].

Infrasound exposure studies in laboratory animals are scarce and report adverse effects in the ear and auditory system [26], brain and central nervous system [27,28], liver [29,30], and lung [31]. Specifically, the

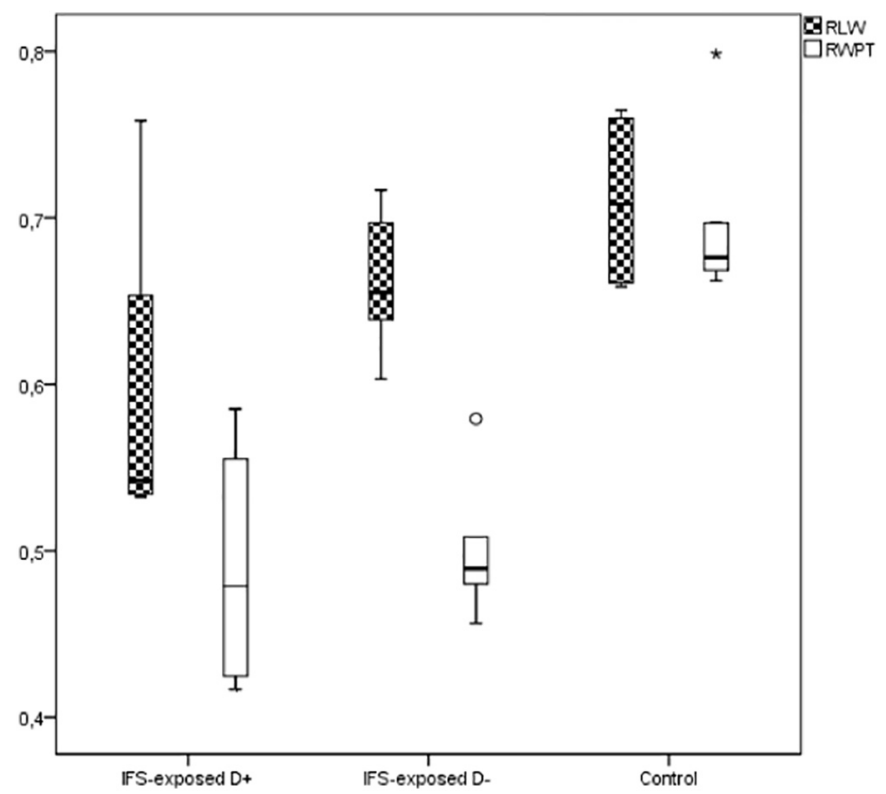

Fig. 4. Lumen-to-vessel wall and vessel wall-to-perivascular tissue ratios in infrasoundexposed dexamethasone-treated rats (group A), infrasound-exposed rats (group B), and control group (group $C$ ). For W/P ratio, there are differences between the groups $(P=$ $.011)$ and between groups $B$ and $C(P=.008)$, but not between groups A and C. RLW, lumen-to-vessel wall ratio; RWP, vessel wall-to-perivascular tissue ratio, D+ and D-, dexamethasone-treated and not treated, respectively. cardiovascular system is sensitive to IFS, as shown by the first studies conducted more than 25 years ago. In these studies, rats were exposed to infrasound $(4,8$, and/or $16 \mathrm{~Hz}$ at 90 to $145 \mathrm{~dB})$ for up to 45 days, which ultimately led to myocardial ischemia and morphofunctional changes in the myocardium cells [32-34]. More recently, Pei et al. reported IFS-induced hemodynamics, cardiac ultrastructure damage, and cardiac cell apoptosis in the rat myocardium [35,36]. The same group found that IFS dysregulates the L-type calcium currents in rat ventricular myocytes [16] and also that acute exposure to IFS induces oxidative damage of cardiomyocytes that affects a series of oxidative damage-related proteins and genes, suggesting a complex signaling network that is evoked by this stressor [37].

There is no agreement about the biological activity of LFN and IFS and the possible underlying mechanisms. The biological effects of noise on living bodies may not be the same due to different parameters such as biological species, frequency, level of sound pressure, or time of exposure. Over the last years, an increased focus from investigators towards the elucidation of these questions has been observed. Increased release of stress hormones, activation of sympathetic nervous system, increased reactive oxygen species production, endothelial dysfunction, peripheral vasoconstriction, increased peripheral vascular resistance, and increased blood viscosity are among the proposed mechanisms elicited by acute or chronic noise stress leading to detrimental outcomes on the cardiovascular system [7,9,38]. Following this line of investigation, Said and El-Gohary studied the effect of noise in the 80-100-dB range on heart rate and mean systemic arterial blood pressure in adult male albino rats and explored possible underlying mechanisms [39]. They concluded that noise stress has many adverse effects on cardiovascular system through increasing plasma levels of stress hormones, oxidative stress, and endothelial dysfunction.

Until recently, it was presumed that LFN required greater sound pressure in order to elicit toxicological effects on humans and animals. High sound pressure levels can be harmful to the cochlea and cause hearing loss, raising the question of other noise effects being secondary, at least partially, to direct auditory damage. Since animal models in previous studies employed mainly high dBA levels (>100-120 dBA), some investigators started exploring the effects of low decibel noise. Jin et al. [17] used isolated and cultured cardiac fibroblasts from rats to study the effects of low decibel IFS. They reported that noise below $90 \mathrm{~dB}$ at $4-20 \mathrm{~Hz}$ inhibits angiotensin-II-stimulated cardiac fibroblasts by reactivating miR-29a targeting the TGF- $\beta /$ Smad3 pathway, possibly eliciting cardiac protective effects. Munzel et al. [18] developed a novel noise exposure model in mice with lower peak sound levels $(<85 \mathrm{dBA})$, lower mean sound pressure levels ( $72 \mathrm{dBA}$ ), and shorter exposure times (1-4 days), thought to cause mainly nonauditory effects to animals such as stress reactions. Exposure to noise resulted in elevated blood pressure and heart rate and was associated with detrimental changes in vascular endothelial function, vascular production of reactive oxygen species, and increased blood stress hormones and biomarkers of inflammation. Notably, they describe an invasion of the vasculature with inflammatory cells. The same group demonstrated that nighttime aircraft noise in healthy volunteers causes endothelial dysfunction, which was partially corrected by the acute administration of vitamin C, pointing to increased oxidative stress as a key mechanism [40].

There are currently limited data on the hypothetical noise-induced pathway involving inflammation [11]. In humans, sleep disturbance is associated with a proinflammatory state [41]. As previously mentioned, the common finding in the noise experiments conducted by our group was the perivascular and myocardial fibrotic development in the absence of inflammatory cells [20-23]. In the present study, we included a group of IFS-exposed animals treated with dexamethasone, a synthetic glucocorticoid member with immunosuppressive potency of about 20-30 times that of hydrocortisone and 4-5 times of prednisone $[42,43]$. Subcutaneous application of dexamethasone, in contrast to intraperitoneal, is highly effective in inhibiting inflammation in mouse models even at low doses [44]. Interestingly, we found differences in 
the comparison of control group with IFS-exposed animals with and without dexamethasone treatment, as the treated animals did not show significant differences when compared to controls. This is the first time that such differences are documented, and despite the absence of inflammatory cells previously described by our group, we have to consider a potential underlying inflammatory mechanism.

The mechanism behind the fibrotic proliferation induced by noise in rat heart is not yet understood. In general, the differentiation of cardiac fibroblasts into more active myofibroblasts is the hallmark of cardiac fibrosis, leading to an abnormal accumulation of the ECM components, such as collagen, around damaged heart tissues [45,46].

Myofibroblast differentiation is a complex and highly regulated process, where biochemical and mechanical factors are interdependent [47]. From a biochemical aspect, the differentiation of cardiac fibroblasts into myofibroblasts is well studied, while the role of mechanical factors remains elusive [48]. When exposed to abnormal mechanical conditions such as strain and ECM stiffness, cardiac fibroblasts can undergo myofibroblast differentiation [49,50]. A fact worth mentioning within the scope of our investigation is that, during the cellular response to heart injury, myofibroblasts actively secrete ECM proteins, such as collagen I and III, to replace the damaged myocardium [51]. We previously performed an immunohistochemical and electron microscopy study in order to evaluate the effects of LFN on cardiac collagen and cardiomyocyte ultrastructure [23]. A significant increase of collagens I and III in the ECM was observed. The ultrastructural observation denoted high concentration of collagen in the ECM next to fibroblasts, confirming the pronounced effect of LFN on the connective tissue.

Comparable to the traditional cardiovascular risk factors, experimental and epidemiological evidence substantiates the concept that noise, through auditory and nonauditory effects, may induce activation of different pathways (oxidative stress, vascular dysfunction, autonomic imbalance) that ultimately lead to cardiac fibrosis, adverse ventricular remodeling, and arrhythmogenesis [7-12]. It is important to note that nonauditory noise effects (annoyance, sleep disturbance, and psychological stress) do not follow the toxicological principle of dosage [7]. Consequently, not simply the accumulated sound energy that causes the adverse effect but also the cognitive perception of the sound, the subsequent cortical activation, and the emotional response need to be taken into account. More epidemiological research on LFN and health effects is needed since the available research is scarce and suffers from methodological shortcomings. A systematic review of observational studies suggests an association between everyday life LFN and IFS components (up to $250 \mathrm{~Hz}$ ) and health effects in the general population, such as annoyance, sleep-related problems, concentration difficulties, and headache [52]. However, they underline the inconsistency across studies and the small number of existing observational investigations, precluding a direct comparison with experimental evidence.

This study has some limitations. The number of animals per group was limited; therefore, the results should be interpreted cautiously. The significant correlation between the two dependent variables considered in this study, ratio L/W and ratio W/P, as expressed by a Spearman correlation coefficient of $0.705(P=.005)$, would recommend a multivariate approach to the data in order to account for the effect of the association between variables on type I error. However, given the reduced dimensions of the groups, it is not recommended to assess the multivariate normality and homogeneity of variance-covariance assumptions in view of the reduced power of the corresponding tests. In these conditions, the Mann-Whitney test has been used to compare the two groups for ratio $\mathrm{L} / \mathrm{W}$ and ratio $\mathrm{W} / \mathrm{P}$ variables, with the Bonferroni correction $\alpha^{*}=0.05 / 2=0.025$. For the reasons mentioned above regarding the correlation between the dependent variables and group dimension, a nonparametric approach to the data was implemented in the comparison between three groups. The Kruskal-Wallis test has been applied with the same Bonferroni correction to the significance level, $\alpha^{*}=0.025$, and post hoc comparisons between the groups were conducted for ratio W/P using the Mann-Whitney test, at the
$0.025 / 3=0.0083$ significance level, to control for inflation of type 1 error. Also, experimental noise stress models are scarce, and at the present time, a well-defined morphological cardiac model to study the consequences of IFS exposure does not exist. There is a lack of consensus regarding the cardiac cell composition, including fibroblasts, in mammals, with potential variations between species that also depend on the age [53]. Concerning the characteristics of noise, public health research uses A-weighting method to measure noise and focus on sound pressure level, disregarding frequencies. We believe that both sound frequency and intensity are key factors. So far, we investigated the structural modifications in the rat myocardium induced by high sound pressure noise of different wavelengths, from industrial to IFS. Addressing these important questions at the mechanistic level in animals may help provide directions for studies in humans, as more epidemiological research is imperative.

\section{Conclusions}

Infrasound exposure induces coronary perivascular fibrosis that differs under corticosteroid administration, which raises the possibility of an underlying inflammatory mechanism. The importance of noise in perturbation of inflammatory factors needs to be further investigated.

\section{Conflicts of interest}

None.

\section{Acknowledgments}

The authors would like to address a posthumous thanks to Professor José Martins dos Santos for contributing to the works related to low frequency noise and infrasound.

\section{References}

[1] Fritschi L, Brown AL, Kim R, Schwela DH, Kephalopoulos S. Burden of disease from environmental noise. World Health Organization; 2011http://www.euro.who.int/_ data/assets/pdf_file/0008/136466/e94888.pdf.

[2] Guski R. Personal and social variables as co-determinants of noise annoyance. Noise Health 1999;1:45-56.

[3] Belojevic G, Jakovljevic B. Factors influencing subjective noise sensitivity in an urban population. Noise Health 2001;4:17-24.

[4] Moller H, Pedersen CS. Hearing at low and infrasonic frequencies. Noise Health 2004;6:37-57.

[5] Heffner HE, Heffner RS. Hearing ranges of laboratory animals. J Am Assoc Lab Anim Sci 2007;46(1):20-2.

[6] Berezhnoy DS, Kiselev NA, Novoseletskaya NM, Inozemtsev AN. Influence of highintensity sound vibration on rat behavior. Moscow Univ Biol Sci Bull 2015;70(2): 53-7.

[7] Babisch W. Cardiovascular effects of noise. Noise Health 2011;13:201-4.

[8] Basner M, Babisch W, Davis A, et al. Auditory and non-auditory effects of noise on health. Lancet 2014;383:1325-32.

[9] Munzel T, Gori T, Babisch W, Basner M. Cardiovascular effects of environmental noise exposure. Eur Heart J 2014;35:829-36.

[10] Munzel T, Sorensen M, Gori T, et al. Environmental stressors and cardio-metabolic disease: part I-epidemiologic evidence supporting a role for noise and air pollution and effects of mitigation strategies. Eur Heart J 2017;38:550-6.

[11] Munzel T, Sorensen M, Gori T, et al. Environmental stressors and cardio-metabolic disease: part II-mechanistic insights. Eur Heart J 2017;38:557-64

[12] Cai Y, Hansell AL, Blangiardo M, et al. Long-term exposure to road traffic noise, ambient air pollution, and cardiovascular risk factors in the HUNT and lifeline cohorts. Eur Heart J 2017;38:2290-6.

[13] Leventhall G. What is infrasound? Prog Biophys Mol Biol 2007;93:130-7.

[14] Ziaran S. The assessment and evaluation of low-frequency noise near the region of infrasound. Noise Health 2014;16:10-7.

[15] Randall JM, Matthews RT, Stiles MA. Resonant frequencies of standing humans. Ergonomics 1997;40:879-86.

[16] Pei Z, Zhuang Z, Xiao P, et al. Influence of infrasound exposure on the whole L-type calcium currents in rat ventricular myocytes. Cardiovasc Toxicol 2009;9:70-7.

[17] Jin W, Deng Q, Chen B, et al. Inhibitory effects of low decibel infrasound on the cardiac fibroblasts and the involved mechanism. Noise Health 2017;19:149-53.

[18] Munzel T, Daiber A, Steven S, et al. Effects of noise on vascular function, oxidative stress, and inflammation: mechanistic insight from studies in mice. Eur Heart J 2017;38:2838-49.

[19] Wu CC, Chen SJ, Yen MH. Effects of noise on blood pressure and vascular reactivities. Clin Exp Pharmacol Physiol 1992;19:833-8. 
[20] Lousinha A, Antunes E, Borrecho G, Oliveira MJ, Brito J, Martins dos Santos J. Histomorphometric evaluation of the small coronary arteries in rats exposed to industrial noise. Int J Mol Sci 2015;16:1095-104

[21] Antunes E, Oliveira P, Oliveira MJ, Brito J, Águas A, Martins dos Santos J. Histomorphometric evaluation of the coronary artery vessels in rats submitted to industrial noise. Acta Cardiol 2013;68:241-5.

[22] Antunes E, Oliveira P, Borrecho G, et al. Myocardial fibrosis in rats exposed to lowfrequency noise. Acta Cardiol 2013:68:285-9.

[23] Antunes E, Borrecho G, Oliveira P, et al. Effects of low-frequency noise on cardiac collagen and cardiomyocyte ultrastructure: an immunohistochemical and electron microscopy study. Int J Clin Exp Pathol 2013;6:2333-41.

[24] Smith SD. Characterizing the effects of airborne vibration on human body vibration response. Aviat Space Environ Med 2002;73:36-45.

[25] Broner N. The effects of low frequency noise on people-a review. J Sound Vib 1978 ; 58:483-500.

[26] Bohne BA, Harding GW. Degeneration in the cochlea after noise damage. Primary versus secondary events. Am J Otol 2000;21:505-9.

[27] Nekhoroshev AS, Glinchikov VV. Effects of infrasound on change in the auditory cortex. Gig Sanit 1992:62-4.

[28] Liu J, Lin T, Yan X, et al. Effects of infrasound on cell proliferation in the dentate gyrus of adult rats. Neuroreport 2010;21:585-9.

[29] Alekseev SV, Glinchikov VV, Usenko VR. Reaction of the liver cells to infrasound. Noise Vib Bull 1987:131-2.

[30] Nekhoroshev AS, Glinchikov VV. Morphological research on the liver structures of experimental animals under the action of infrasound. Aviakosm Ekolog Med 1992:26:56-9.

[31] Svidovyi VI, Glinchikov VV. Action of infrasound on the lung structure. Noise Vib Bull 1987:153-4.

[32] Alekseev SV, Glinchikov VV, Usenko VR. Myocardial ischemia in rats exposed to infrasound. Gig Tr Prof Zabol 1983;8:34-8.

[33] Gordeladze AS, Glinchikov VV, Usenko VR. Experimental myocardial ischemia caused by infrasound. Gig Tr Prof Zabol 1986;6:30-3.

[34] Nekhoroshev AS, Glinchikov VV. Morpho-functional changes in the myocardium after exposure to infrasound. Gig Sanit 1991;12:56-8.

[35] Pei Z, Sang H, Li R, et al. Infrasound-induced hemodynamics, ultrastructure, and molecular changes in the rat myocardium. Environ Toxicol 2007;22:169-75.

[36] Pei ZH, Chen BY, Tie R, et al. Infrasound exposure induces apoptosis of rat cardiac myocytes by regulating the expression of apoptosis-related proteins. Cardiovasc Toxicol 2011;11:341-6.

[37] Pei Z, Meng R, Zhuang Z, et al. Cardiac peroxisome proliferator-activated receptor-y expression is modulated by oxidative stress in acutely infrasound-exposed cardiomyocytes. Cardiovasc Toxicol 2013;13:307-15.
[38] Baldwin AL, Wagers C, Schwartz GE. Reiki improves heart rate homeostasis in laboratory rats. J Altern Complement Med 2008;14:417-22.

[39] Said M, El-Gohary O. Effect of noise stress on cardiovascular system in adult male albino rat: implication of stress hormone, endothelial dysfunction and oxidative stress. Gen Physiol Biophys 2016;35:371-7.

[40] Schmidt FP, Basner M, Kroger G, et al. Effect of nighttime aircraft noise exposure on endothelial function and stress hormone release in healthy adults. Eur Heart J 2013; 34:3508-14.

[41] Irwin MR, Olmstead R, Carrol JE. Sleep disturbance, sleep duration, and inflammation: a systematic review and meta-analysis of cohort studies and experimental sleep deprivation. Biol Psychiatry 2016;80:40-52.

[42] Rhen T, Cidlowski JA. Anti-inflammatory action of glucocorticoids-new mechanisms for old drugs. N Engl J Med 2005;353:1711-23.

[43] Mager DE, Moledina N, Jusko WJ. Relative immunosuppressive potency of therapeutic corticosteroids measured by whole blood lymphocyte proliferation. J Pharm Sci 2003;92:1521-5

[44] Weichhart T, Brandt O, Lassnig C, et al. The anti-inflammatory potency of dexamethasone is determined by the route of application in vivo. Immunol Lett 2010;129: $50-2$.

[45] Borne SW, Diez J, Blankesteijn WM, Verjans J, Hofstra L, Narula J. Myocardial remodeling after infarction: the role of myofibroblasts. Nat Rev Cardiol 2010;7:30-7.

[46] Wynn TA, Ramalingam TR. Mechanisms of fibrosis: therapeutic translation for fibrotic disease. Nat Med 2012;18:1028-40.

[47] MacKenna D, Summerour SR, Villarreal FJ. Role of mechanical factors in modulating cardiac fibroblast function and extracellular matrix synthesis. Cardiovasc Res 2000; 46:257-63.

[48] Yong KW, Li Y, Huang G, et al. Mechanoregulation of cardiac myofibroblast differentiation: implications for cardiac fibrosis and therapy. Am J Physiol Heart Circ Physiol 2015;309:H532-42.

[49] Costa AP, Clemente CF, Carvalho HF, Carvalheira JB, Nadruz W, Franchini KG. FAK mediates the activation of cardiac fibroblasts induced by mechanical stress through regulation of the mTOR complex. Cardiovasc Res 2010;86:421-31.

[50] Hinz B. The myofibroblast: paradigm for a mechanically active cell. J Biomech 2010; 43:146-55.

[51] Ma Y, Bras LE, Toba H, et al. Myofibroblasts and the extracellular matrix network in post-myocardial infarction cardiac remodeling. Pflügers Arch 2014;466:1113-27.

[52] Baliatsas C, van Kamp I, van Poll R, Yzermans J. Health effects from low-frequency noise and infrasound in the general population: is it time to listen? A systematic review of observational studies. Sci Total Environ 2016;557-8:163-9.

[53] Pinto AR, Ilinykh A, Ivey MJ, et al. Revisiting cardiac cell composition. Circ Res 2016; $118: 400-9$. 